\title{
Fibrodisplasia Osificante Progresiva: Reporte de Caso.
}

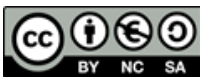

\author{
Fibrodysplasia Ossificans Progressiva: Case report.
}

\author{
Pavel Amigo Castañeda. ${ }^{1}$, Maylin Rodríguez Díaz. ${ }^{2}$, Caridad María Castañeda \\ Gueimonde. ${ }^{3} \&$ Pavel Alejandro Amigo Rodríguez. ${ }^{4}$
}

Recibido: 07-02-2021 / Revisado: 16-02-2021 /Aceptado: 07-03-2021/ Publicado: 05-04-2021

\section{DOI: https://doi.org/10.33262/anatomiadigital.v4i2.1619}

\section{Summary.}

Introduction: Fibrodysplasia Ossificans Progressiva is a rare, severely disabling autosomal dominant disease. In most patients it is due to a new mutation in previously unaffected families. It is characterized by progressive heterotopic ossification of connective tissue, aponeurosis, fascia, ligaments, tendons, and skeletal muscle. Early diagnosis improves the prognosis and quality of life despite the fact that there is no definitive treatment, so the objective is to show the results of a patient admitted and studied with the diagnosis of Fibrodysplasia Ossificans Progressiva. Methodology A descriptive, cross-sectional study is carried

\section{Resumen.}

Introducción: La Fibrodisplasia Osificante Progresiva es una enfermedad rara de transmisión autosómica dominante severamente incapacitante. En la mayoría de pacientes se debe a una mutación nueva en familias no afectadas previamente. Se caracteriza por osificación heterotópica progresiva del tejido conectivo, aponeurosis, fascia, ligamentos, tendones y músculo esquelético. El diagnóstico precoz mejora el pronóstico y la calidad de vida a pesar que no existe tratamiento definitivo, por lo que el objetivo constituye mostrar los resultados de un paciente ingresado y estudiado con el diagnóstico de Fibrodisplasia Osificante Progresiva. Metodología Se realiza un

\footnotetext{
1 Hospital Pediátrico Eliseo Noel Caamaño, Matanzas, Cuba, reynaldoamigo.mtz@infomed.sld.cu (iD) https://orcid.org/ 0000-0003-3015-9269

2 Hospital Pediátrico Eliseo Noel Caamaño, Matanzas, Cuba. maylinrd.mtz@infomed.sld.cu (iD) https://orcid.org/ 0000-0002-6600-7403

3 Hospital Faustino Pérez Hernández, Matanzas, Cuba. caridadcastaneda.mtz@infomed.sld.cu (iD https://orcid.org/ 0000-0003-3952-5181

4 Universidad de Ciencias Médicas de Matanzas, Facultad de Ciencias Médicas Juan Guiteras Gener. Matanzas, Cuba, pavelamigorodriguez@gmail.com (D) https://orcid.org/ 0000-0003-3596-0637
} 
out, where the results obtained through the study of a patient with a diagnosis of Fibrodysplasia Ossificans Progressiva are shown at the Elíseo Noel Camaño Provincial Pediatric Hospital of the Province of Matanzas in the period of November 2019. Results: The evolution and results obtained in the study are shown performed on a 4-year-old patient where the diagnosis of a rare disease called Fibrodysplasia Ossificans Progressiva. Conclusions: a more serious cause of heterotopic ossification in skeletal muscles, fasciae, tendons and ligaments without effective treatment and that evolves by pushing.

Keywords: Fibrodysplasia Ossificans Progressiva; Heterotopic ossification. estudio descriptivo, transversal, donde se muestra los resultados obtenidos mediante el estudio de un paciente con diagnóstico de Fibrodisplasia Osificante Progresiva, en el Hospital Provincial Pediátrico Elíseo Noel Camaño de la Provincia de Matanzas en el periodo de noviembre del 2019. Resultados. Se muestra la evolución y los resultados obtenidos en el estudio realizado a una paciente de 4 años de edad donde se concluye el diagnóstico de una rara enfermedad que se denomina Fibrodisplasia Osificante Progresiva. Conclusiones: es la causa más grave de osificación heterotópica en músculos esqueléticos, fascias, tendones y ligamentos sin tratamiento eficaz y que evoluciona por empujes.

\section{Palabras}

Claves: Fibrodisplasia

Osificante Progresiva;

Osificación heterotópica.

\section{Introducción.}

La Fibrodisplasia Osificante progresiva es una enfermedad genética del tejido conectivo de escasa prevalencia, hereditaria, severamente rara e incapacitante, que ha afectado hasta la fecha a 2,500 personas aproximadamente en el mundo y aún no se ha encontrado tratamiento definitivo. Se han reportado en España alrededor de 26 pacientes con el diagnóstico de FOP hasta el 2018. (Salinas, 2018) La primera mención de casos de Fibrodisplasia Osificante Progresiva se remonta a 1692 por Guy Patin y luego, Frake en 1739. En 1924 Noble propuso dividirla en tres categorías: Miositis Osificante progresiva, Miositis Osificante circunscrita progresiva y Miositis Osificante circunscrita sin antecedentes de traumatismo. Fue en 1969 cuando Munchmever describió la enfermedad. (Aren, 2014)

Es una enfermedad ocasionada por un gen que codifica el receptor de la proteína morfogenética ósea, denominado Activin tipo 1 o ACVR1 del cual cada célula tiene dos copias de dicho gen y en alguna se encuentra la mutación. Se produce hueso nuevo no neoplásico en lugares donde no debe existir. (Kaplan, 2012).

La incidencia se estima en 1 portador por cada dos millones de nacidos vivos. El $75 \%$ de la población afectada presenta deformidades que ayudan al reconocimiento de la 
enfermedad: Hallux Valgus congénito, amplios cuellos femorales, metacarpianos cortos, poca capacidad pulmonar, ligero retraso mental. Ocurre en la segunda o tercera década de la vida; se localiza comúnmente en los músculos cuádriceps y braquial, y en menor frecuencia en deltoides, región escapular y mano. Respeta los músculos faciales, la lengua, el diafragma, los músculos de la pared abdominal y el músculo liso de las víseras. (Salinas, 2018, Pignolo, 2013).

Se presenta con dolor súbito de una a dos semanas después de traumatismos leves, con edema, aumento de la temperatura en la región afectada, disminución de los arcos de movimiento, aumento de la fosfatasa alcalina y de la velocidad de sedimentación globular. El dolor disminuye de forma progresiva.

La reacción inflamatoria muscular producida por traumatismos menores, como el uso de agujas hipodérmicas en tratamientos odontológicos, inyecciones intramusculares, vacunas, fracturas, contusiones, etc, llevan a un proceso de osificación del tejido conectivo y muscular y que en casos graves cuando afecta los músculos respiratorios, produce Insuficiencia respiratoria restrictiva. Este estudio se justifica, en opinión de los autores pues el diagnóstico tardío de esta enfermedad debido a su baja incidencia y la falta de conocimiento del personal de salud, empeora el pronóstico, por lo que nos trazamos como objetivo mostrar los resultados de un paciente ingresado y estudiado con el diagnóstico de Fibrodisplasia Osificante Progresiva.

\section{Metodologia.}

Se realiza la presentación de un caso interesante, donde se muestran los resultados obtenidos mediante el estudio de dicho paciente con diagnóstico de Fibrodisplasia Osificante Progresiva, en el Hospital Provincial Pediátrico Elíseo Noel Caamaño de la provincia de Matanzas, durante el año 2019.

\section{Resultados.}

Presentación del caso: Paciente femenina de 4 años de edad, originaria de la barriada de Pueblo Nuevo en la ciudad de Matanzas, sin antecedentes heredofamiliares de importancia. Inició su padecimiento dos meses previos al ingreso en el Hospital Pediátrico de Matanzas tras caer de sus pies durante el juego y sufrir contusiones en los miembros inferiores, por lo que a partir de ese momento comenzó a sentir y a referir además de constatarlo, trastornos progresivos para la marcha y dolor muscular. Presentó edema, aumento de temperatura y dolor a la palpación en ambos miembros inferiores. Días después se añadieron parestesias e incapacidad para la deambulación, por lo que se prescribió un medicamento (no especificado por el familiar), sin notar mejoría. Con el aumento del dolor se le realizan estudios de Rx y Ultrasonidos de partes blandas, así como estudios de hematología. Se discute en colectivo con Pediatría, Neurología, Fisiatría y se observó a una paciente femenina, de edad biológica similar a la cronológica, sin facies característica, dedos de ambas manos cortos y gruesos, con incapacidad para la deambulación, con limitación a la flexo-extensión, aumento de la temperatura en los muslos del tercio distal, no presentaba déficit cognitivo, ni tenía antecedentes familiares de Fibrodisplasia Osificante Progresiva. 
El examen neurológico mostró un paciente despierto, consciente, orientado, con respuestas adecuadas al interrogatorio. Balance motor, sensibilidad y reflejos osteotendinosos normales. Respuesta plantar flexora bilateral. En ambos miembros inferiores aumento del tono muscular en la cara anterior y región glútea del lado derecha, sin datos de infección o sangrado. El miembro derecho con aumento de tono hasta el tercio proximal de la pierna. Se conservan los arcos de movimiento en la articulación tibio-astragalina, pero no así los de la rodilla derecha, pulsos distales sin alteraciones, sin datos de afectación neurovascular distal. Pulso poplíteo derecho no palpable a expensas de induración a nivel de la región poplítea de ese miembro. En las radiografías de ambos muslos y pelvis se observaban extensas bandas y calcificaciones de tipo en rosario en ambos muslos, que se extendían hasta la región de los trocánteres, además de visualizarse una zona radiopaca de forma lineal a nivel de región posterior de rodilla derecha. Los exámenes de laboratorio reportaron $\mathrm{Hb}$ 11,2, Leucocitos 7,4, Plaquetas 255, Fosfatasa Alcalina de 673, Factor Reumatoide (+), PCR (-), Perfil Tiroideo normal.

Se indicó tratamiento con esteroides, los síntomas remitieron, pero no la incapacidad física (falta de deambulación); tampoco desaparecieron las zonas engrosadas de ambos miembros inferiores, específicamente a ambos lados de los muslos ni región poplítea por lo que se decide tomar un pequeña muestra de ese tejido con el objetivo de esclarecer diagnóstico por lo que se selecciona el área que menos afectación pudiera ocasionar en caso de que la fibrosis pudiera progresar por la agresión de la actividad quirúrgica. Se toma la muestra del muslo derecho en su cara externa y, sin embargo, se decide su egreso de la unidad por mejoría clínica y en espera del resultado de la biopsia. A los 30 días la biopsia informa tejido óseo heterotópico a ese nivel por lo que en conjunto con Pediatría y Genética se concluye el diagnóstico de Fibrodisplasia Osificante progresiva.

Fig.1. Imagen radiológica con bandas y calcificaciones de tipo en rosario a nivel del muslo.

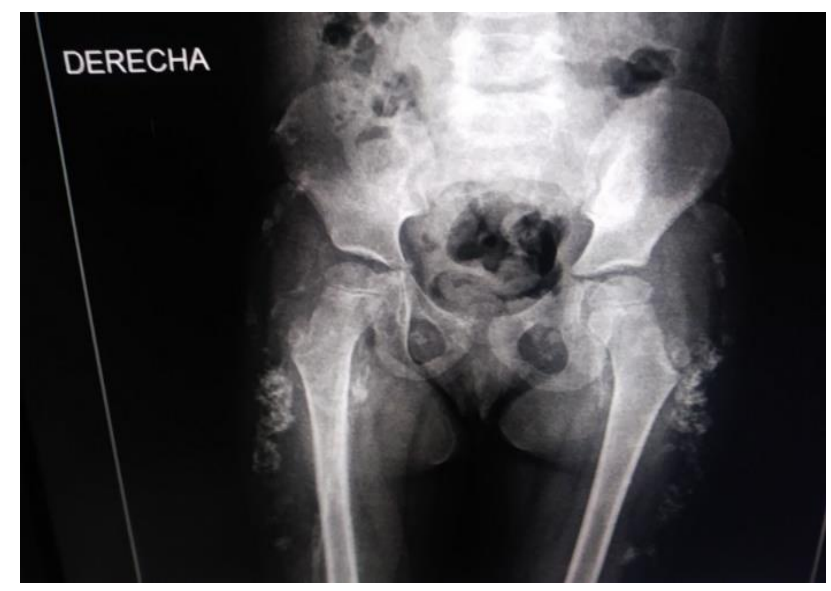

Fuente: Elaboración propia. 
Fig.2. Imagen radiológica AP con bandas y calcificaciones de tipo en rosario a nivel del muslo.

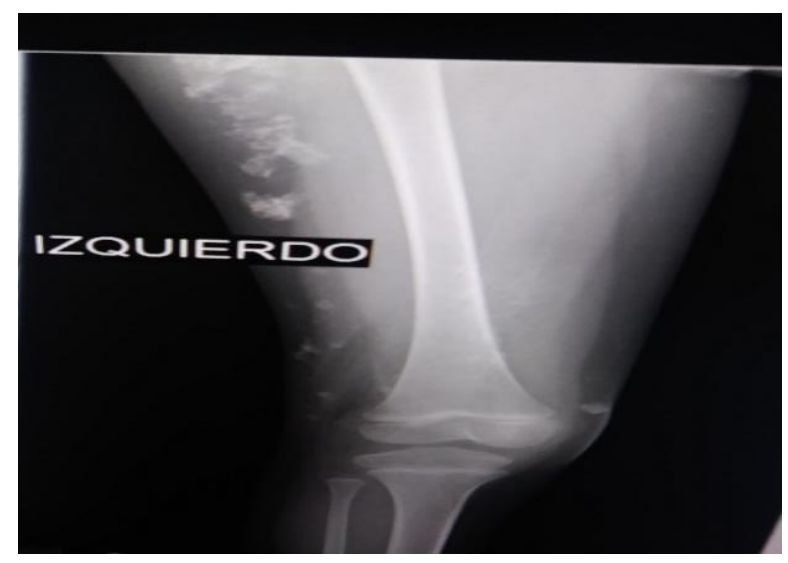

Fuente: Elaboración propia.

Fig.3. Imagen Radiológica lateral de lesiones radiopacas a nivel de tercio inferior del muslo.

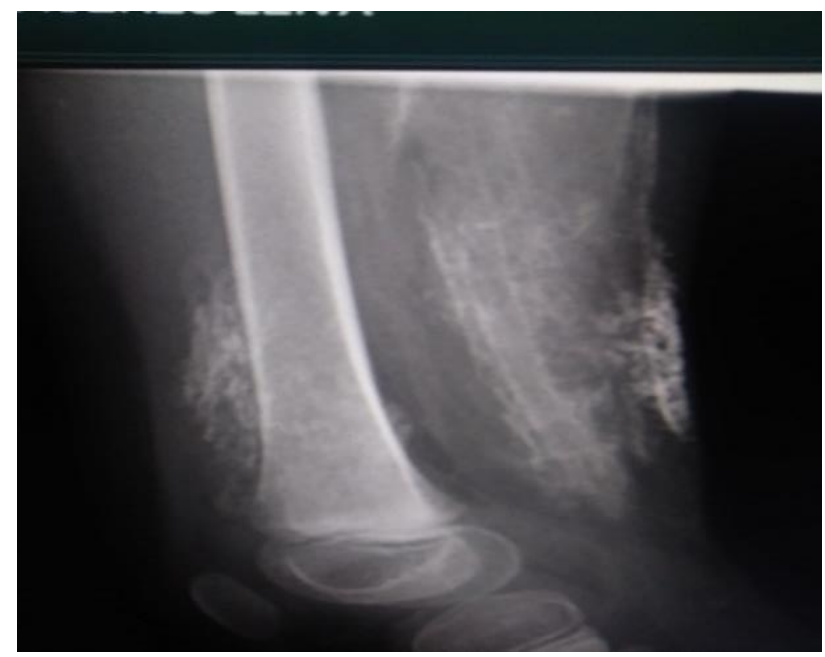

Fuente: Elaboración propia.

Fig.4. Imagen Radiológica AP de lesiones radiopacas a nivel de tercio inferior del muslo.

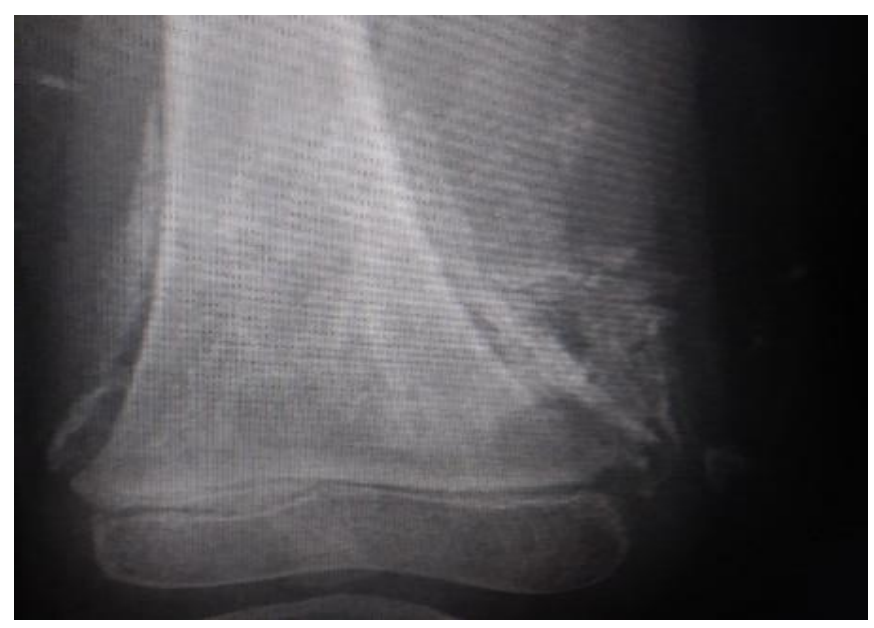

Fuente: Elaboración propia. 


\section{Discusión de los resultados.}

La Fibrodisplasia Osificante progresiva (FOP) es una enfermedad genética, rara, caracterizada por osificación heterotópica endocondral. Se debe a una mutación genética que aparece cuando se forma el gameto, no se sabe si por un cambio por parte del padre o de la madre. Se produce un error de forma que la proteína encargada de producir hueso, que debería estar inactiva, permanece continuamente activada. No hay preferencia por ninguna raza y afecta por igual a hombres y mujeres. La enfermedad generalmente inicia en la primera década de vida con episodios acumulativos de dolor, edema e inflamación de músculos, aponeurosis, fascia, ligamentos y tendones, desencadenados por traumas como inyecciones intramusculares, caídas, biopsias y procedimientos odontológicos entre otros. (Pignolo, 2013, Morales, 2010)

EL acortamiento del dedo gordo del pie en forma bilateral por hipoplasia de la segunda falange es característico y puede ser observado desde el nacimiento. Este fenotipo podría permitir un diagnóstico temprano, aun antes de la aparición de focos de inflamación, dolor muscular y osificación endocondral heterópica. (Morales 2010, Kitterman 2005) El 90 \% de pacientes con FOP no han sido diagnosticados adecuadamente sino hasta un período avanzado de la enfermedad. (Kitterman, 2005) Esto lleva a realización de exámenes invasivos, aplicación de inyecciones intramusculares, toma de muestras de laboratorio, procedimientos odontológicos, biopsias y otros procedimientos que causan lesión tisular y el empeoramiento del cuadro. (Kitterman, 2005, Kaplan, 2008) El conocimiento adecuado de esta enfermedad pudo haber permitido un diagnóstico temprano y así evitado algunos procedimientos invasivos y caídas que les provocaron episodios de inflamación aguda y osificación progresiva con el consecuente aumento de la discapacidad.

La mayoría de pacientes con FOP resultan de una mutación espontánea. Cuando se observa transmisión genética es autosómica dominante. La patología molecular primaria compromete la proteína morfogénica del hueso. (Kaplan, 2010, Hegyi, 2003, Kaplan, 2011) En cuanto a su prevalencia no está descrita en todos los países, pero en los que se ha estudiado se da tanto en hombres como en mujeres. En 2006 se descubrió cuál era la mutación genética y a partir de ahí se ha avanzado mucho en su conocimiento. Estos enfermos tienen una característica: nacen con una deformidad en los pies llamada Hallux Valgus o juanete. Y para la confirmación se tiene ya disponible el estudio genético. Aun así, es una enfermedad rara y muchas veces en atención primaria no se piensa en ella. Se ha comunicado una elevada frecuencia de síntomas neurológicos crónicos.

Es así como la enfermedad empieza a manifestarse, con inflamaciones que suelen arrancar en la primera década de la vida, por una caída o de forma espontánea, y que provocan que el tejido muscular de la zona se convierta en hueso. Los brotes se van extendiendo hasta que la rigidez es absoluta. Una vez que el músculo se ha osificado, los pacientes no sienten dolor, pero en el momento de la hinchazón, los huesos surgen con pinchos. Comúnmente los denominan "espinas de rosal", lo que puede dar una idea del sufrimiento que provocan. Los datos radiográficos aparecen de dos a cuatro semanas después de la lesión. (Salinas, 
2018, Aren, 2014, Kravitz, 2011) El diagnóstico es clínico y radiológico y se observa la evolución del paciente y la formación de hueso extraóseo. Un brote de Fibrodisplasia Osificante progresiva puede durar de seis a ocho semanas, en tanto que la rigidez de las articulaciones puede ser de un momento a otro y sólo presentar dolor incapacitante con aumento de fosfatasa alcalina. A menudo la intervención quirúrgica empeora la condición porque crece un hueso nuevo en el sitio de la lesión, que daña aún más la movilidad. Se tiene que realizar diagnóstico diferencial de cáncer, fibromatosis infantil agresiva, displasia fibrosa y heteroplasia ósea progresiva. En este paciente disminuyó el dolor, pero no la incapacidad física.

La osificación heterotópica con frecuencia inicia a nivel dorsal, craneal, axial y regiones proximales de las extremidades. Posteriormente la enfermedad compromete regiones ventrales, apendiculares y distales. No hay compromiso de diafragma, lengua y músculos extra oculares. (Pignolo, 2013, Kaplan, 2011, Avila, 2009) Los episodios, que son acumulativos, le llevan al paciente a silla de ruedas alrededor de la tercera década. (Pignolo, 2013, Kravitz 2011, Pignolo, 2011) La complicación más importante es el síndrome de insuficiencia respiratoria restrictiva por rigidez del tórax que puede complicarse con neumonía y falla cardiaca derecha. Otras complicaciones son la anquilosis de la articulación temporo - mandibular desencadenado por manipulación odontológica y sordera por osificación del oído medio. (Kravitz, 2011, Avila, 2009, Lakkireddy, 2015) Las complicaciones pueden ser prevenidas, por lo que es importante la educación médica para el diagnóstico temprano de esta enfermedad.

Estudios realizados reportan fallecimientos entre los grupos de edades de 40 a 50 años. El tórax, que debe moverse para que entre y salga aire, acaba afectado por lo que suelen tener problemas respiratorios e infecciones y una muerte temprana por ese motivo. Se plantea que se está empezando a ver una luz al final del túnel en cuanto al tratamiento medicamentoso, pues los tratamientos en estudio si bien no devuelven los tejidos dañados a su estado natural si previenen las inflamaciones, logran evitar nuevos episodios o que sean menos agresivos y evitan que la enfermedad progrese, de ahí la importancia del diagnóstico temprano. Estos ya están en fase de ensayo clínico, lo que significa que se están probando en pacientes. El primero, en el que participa el Hospital La Fe de Valencia, se focaliza en niños y el segundo, en el que interviene el Ramón y Cajal de Madrid, se ha dirigido a adultos. (Aren, 2014, Akesson, 2020)

Aunque el mejor tratamiento sigue siendo el preventivo. Se debe evitar caídas, biopsias e inyecciones intramusculares. Es recomendable realizar profilaxis de caries para evitar tratamientos odontológicos agresivos. (Avila, 2009, Choquet, 2017, Kaplan, 2019, Morales, 2014)

\section{Conclusiones.}

- La Fibrodisplasia Osificante progresiva es una enfermedad de la que no se sabe cuándo inicia debido a su rareza; es de difícil diagnóstico, el cual es clínico y 
radiológico; no hay datos de laboratorio o patrón de referencia que oriente hacia el padecimiento.

- Es una enfermedad incapacitante que empeora con el tiempo y hasta el día de hoy no hay tratamiento alguno que limite el daño.

\section{Referencias bibliográficas.}

Akesson LS., Savarirayan R., (2020). Fibrodysplasia Ossificans Progressiva. Jun 11. In: Adam MP, Ardinger HH, Pagon RA, Wallace SE, Bean LJH, Stephens K,Amemiya A, Editors. GeneReviews ${ }^{\circledR}$ [Internet]. Seattle (WA): University of Washington, Seattle; 1993-2020. PMID: 32525643.

Aren Frontera. J., López Garrido J., (2014). Manejo del dolor en la Fibrodisplasia Osificante Progresiva. Revista de la Sociedad Española del dolor Versión impresa ISSN1134- $8046 \quad$ Vol.21 No.5 Madrid sep. /oct. http://dx.doi.org/10.4321/51134-80462014000500004.

Avila Saldívar AC., Guerrero V., (2009). Fibrodisplasia Osificante progresiva. Reporte de Un Caso y revisión de la Bibliografía. Medicina Interna de México Volumen 25, núm. 3, mayo-junio 245 Med Int Mex; 25 (3):245-7. Disponible en http://www.revistasmedicas.com.mx

Choquet R., Bouee S., Jeanbat V., et al. (2017). Prevalence of fibrodysplasia ossificans Progressiva (FOP) in France: an estimate based on a record linkage of two national Databases. Orphanet J Rare Dis.; 12:123.

Hegyi L., Gannon FH. Glaser DL. Et al. (2003). Stromal cells of fibrodysplasia ossificans Progressiva lesions express smooth muscle lineage markers and the osteogenic Transcription factor Runx2/Cbfa-1: clues to a vascular origin of heterotopic Ossification? J Pathol 2003; 201:141-8. [ [ Links ]

Kaplan FS., Al Mukaddam M., Baujat G., et al (2019). The medical management of Fibrodysplasia ossificans Progressiva: current treatment considerations. Proc Intl Clin Council FOP.; 1:1-111. Available http://www.iccfop.org/dvlp/wpcontent/uploads/2020/03/GuidelinesJanuary

Kaplan F.S., Shore E.M., Pignolo RJ. (2011). The medical management of fibrodysplasia Ossificans progressiva: Current treatment considerations. Clin Proc Intl Clin Consort FOP; 4:1-100.

Kaplan FS., Le Merrer M., Glaser DL., et al. (2008). Fibrodysplasia ossificans progressiva. Best Pract Res Clin Rheumatol. 22:191-205. [ L Links ]

Kaplan FS., Zasloff MA., Kitterman JA., et al (2010). Early mortality and cardiorespiratory Failure in patients with fibrodysplasia ossificans progressiva. J Bone Joint Surg Am; 92:686-91. [ [ Links ] 
Kaplan F.S., CHakkalakal S.A., Shore E.M., (2012). Fibrodysplasia ossificans progressiva: Mechanisms and models of skeletal metamorphosis Dis Model Mech; 5(6):756-62.

Kitterman JA., Kantanie S., Rocke DM. Kaplan FS. (2005). Iatrogenic harm caused by Diagnostic errors in fibrodysplasia ossificans progressiva. Pediatrics. 116: e65461. Obtenido de http://pediatrics.aappublications.org/content/116/5/e654 [ Links ]

Kravitz J., Dominici P Ufberg J., Fisher J., et al. (2011). Two Days of dexamethasone Versus 5 days of prednisone in the treatment of acute asthma: A randomized controlled trial. Ann Emerg Med: 58 (2): 200-4.

Lakkireddy M., Chilakamarri V., Ranganath P., et al. (2015). Clinical and Genetic Analysis of Fibrodysplasia Ossificans Progressiva: A Case Report and Literature Review.J Clin Diagn Res. Aug; 9(8): RD01-3. Doi: 10.7860/JCDR/2015/15160.6393. Epub 2015 Aug 1. PMID: 26436010 Free PMC article.

Morales-Piga A., Kaplan FS., (2010). Osteochondral diseases and fibrodysplasia ossificans Progressiva. Adv Exp Med Biol 686:335-48. Obtenido de https://www.ncbi.nlm.nih.gov/pubmed/20824454 [ $\underline{\text { Links }] ~}$

Morales-Pigaa M., Bachiller-Corralb F J., Sánchez-Duffhuesc G. (2014). ¿Es la Fibrodisplasia Osificante progresiva una enfermedad de origen vascular? Un modelo Patogénico innovador Reumatol Clin.; 10(6):389-395

Obtenido de http://www.reumatologiaclinica.org

Pignolo RJ., Shore EM., Kaplan FS., (2013). Fibrodysplasia ossificans progressiva: diagnosis, Management and therapeutic horizons. Rev Pediatr Endocrinol. 10 Suppl 2:437-48. Obtenido de https://www.ncbi.nlm.nih.gov/pubmed/23858627 [ Links ]

Pignolo RJ., Shore EM., Kaplan FS., (2011). Fibrodysplasia ossificans progressiva: Clinical and genetic aspects. Orphanet J Rare Dis. Dec 1; 6:80. Doi: 10.1186/1750-1172-6-80. PMID: 22133093 Free PMC article. Review.

Salinas-Suikouski. R., Saltos-Arauz J., (2018). Fibrodisplasia Osificante Progresiva: Reporte De Casos. Revista Ecuatoriana de Neurología versión On-line ISSN 2631-2581 Versión impresa ISSN 1019-8113 vol.27 no.2 Guayaquil May. /ago.

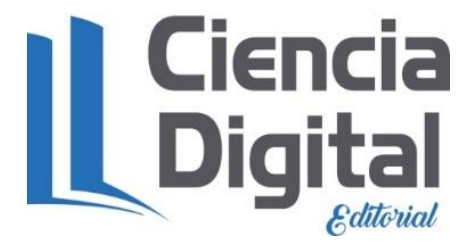


PARA CITAR EL ARTÍCULO INDEXADO.

Amigo Castañeda, P., Rodríguez Díaz, M., Castañeda Gueimonde, C. M., \& Amigo Rodríguez, P. A. (2021). Fibrodisplasia Osificante Progresiva: Reporte de Caso . Anatomía Digital, 4(2), 78-87. https://doi.org/10.33262/anatomiadigital.v4i2.1619

\section{Ciencia
Digital
Edtatoal}

El artículo que se publica es de exclusiva responsabilidad de los autores y no necesariamente reflejan el pensamiento de la Revista Anatomía Digital.

El artículo queda en propiedad de la revista y, por tanto, su publicación parcial y/o total en otro medio tiene que ser autorizado por el director de la Revista Anatomía Digital.
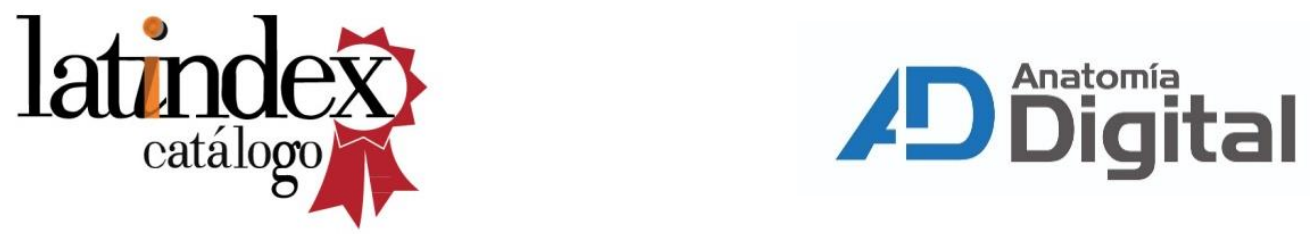